Control/Tracking Number: 15-SA-3737-ACSM

Activity: Scientific Abstract

Current Date/Time: 11/11/2014 7:37:05 AM

\title{
The Effects Of A Pilates Intervention On Arterial Stiffness And Trunk Flexibility
}

Author Block: Maria Konstantaki, Jessica Fasbender, Tom Cudmore. Buckinghamshire New University, High Wycombe, United Kingdom.

\begin{abstract}
:
Arterial stiffness has been identified as an independent risk factor for cardiovascular disease, whereas low trunk flexibility has been suggested to be a predictor of arterial stiffening. Pilates has been shown to improve trunk flexibility in middle aged women however, the effect of Pilates on arterial compliance has not been investigated.

PURPOSE: To determine the effect of a Pilates intervention on arterial compliance and trunk flexibility.

METHODS: Twenty participants (age range: 24-61 years) were assigned into either Group A: physically active individuals with previous Pilates experience (PEPA, $n=8$ ) or Group $B$ : individuals without previous Pilates experience and/or physically inactive (NPE-LPA, $n=12$ ). Both groups participated in two 55-minute Pilates sessions per week for six weeks. In addition, all subjects performed a 10-min home programme twice a week in between sessions. Augmentation index (Al; $\%$ ), peripheral (pSys and pDia; $\mathrm{mmHg}$ ) and central blood pressures (cSys and cDia; $\mathrm{mmHg}$ ) were recorded using a vascular testing device. Trunk flexibility index was calculated by adding the scores from a trunk flexion (sit-and-reach test) and a trunk extension test. All measurements were conducted at baseline and immediately after the six week intervention. RESULTS: In the PEPA group, there were reductions in all blood pressures post-intervention (pSys: $106 \pm 7 \mathrm{mmHg} v 102 \pm$ $6 \mathrm{mmHg}$; $=0.011$; pDia: $68 \pm 6 \mathrm{mmHg} \vee 65 \pm 4 \mathrm{mmHg}, \mathrm{p}=0.015$; cSys: $100 \pm 9 \mathrm{mmHg} \vee 95 \pm 7$ $\mathrm{mmHg} ; \mathrm{p}=0.016$; cDia: $69 \pm 6 \mathrm{mmHg} \vee 67 \pm 4 ; \mathrm{p}=0.029$ ). In the NPE-LPA group, significant decreases were noted post-intervention in Al (pre: $81 \pm 46 \%$ and post: $59 \pm 33 \% ; p=0.014$ ) and cSys (pre: $103 \pm 13 \mathrm{mmHg}$ and post: $99 \pm 9 \mathrm{mmHg} ; p=0.049$ ). Significant decreases in trunk flexibility were observed in both the PEPA $(89.3 \pm 17 \mathrm{~cm} v 95.4 \pm 16.1 \mathrm{~cm} ; \mathrm{p}=0.001)$ and NPE-LPA group $(70 \pm 24.2 \mathrm{~cm} \vee 76.5 \pm 20.2 \mathrm{~cm} ; p=0.026)$. A link between poor trunk flexibility and arterial stiffness was observed, but was not statistically significant ( $p>0.05)$. CONCLUSION: Findings suggest that Pilates is effective for reducing arterial stiffness and/or maintaining the elastic properties of the arteries. The exact mechanisms could not be associated with increases in flexibility in this study. It might be that increases in muscle strength play a role, however further research is needed to acsertain this relationship.
\end{abstract}

1995 characters

Author Disclosure Information: M. Konstantaki: None.

Category (Complete): 1200. Exercise is Medicine - Focuses on the impact of physical activity on health and the prevention and treatment of disease and disability in clinical settings

Keyword (Complete): arterial stiffness ; trunk flexibility ; Pilates

Unlabeled/ Investigational Products (Complete):

: No

Presentation Preference (Complete): Exercise is Medicine - Poster

Area of Interest (Complete):

Area of Interest: Applied Science 